\title{
ON THE EXTENDED HAAGERUP TENSOR PRODUCT IN OPERATOR SPACES
}

\author{
TAKAShi Itoh AND MASARU Nagisa
}

\begin{abstract}
We describe the Haagerup tensor product $\ell^{\infty} \otimes_{h} \ell^{\infty}$ and the extended Haagerup tensor product $\ell^{\infty} \otimes_{e h} \ell^{\infty}$ in terms of Schur product maps, and show that $\ell^{\infty} \otimes_{h} \ell^{\infty} \cap \mathbb{B}\left(\ell^{2}\right)$ (resp. $\left.\ell^{\infty} \otimes_{e h} \ell^{\infty} \cap \mathbb{B}\left(\ell^{2}\right)\right)$ coincides with $c_{0} \otimes_{h} c_{0} \cap \mathbb{B}\left(\ell^{2}\right)$ (resp. $\left.c_{0} \otimes_{e h} c_{0} \cap \mathbb{B}\left(\ell^{2}\right)\right)$. For $\mathrm{C}^{*}$-algebras $A, B$, it is shown that $A \otimes_{h} B=A \otimes_{e h} B$ if and only if $A$ or $B$ is finite-dimensional.
\end{abstract}

\section{Introduction}

For Hilbert spaces $\mathcal{H}$ and $\mathcal{K}$, we let $\mathbb{B}(\mathcal{H}, \mathcal{K})$ and $\mathbb{K}(\mathcal{H}, \mathcal{K})$ denote the bounded operators and the compact operators of $\mathcal{H}$ to $\mathcal{K}$. An operator space $X$ on $\mathcal{H}$ is a subspace of $\mathbb{B}(\mathcal{H})=\mathbb{B}(\mathcal{H}, \mathcal{H})$ which is endowed with norms to each $n \times m$ matrices $\mathbb{M}_{n, m}(X)$ over $X$ as a subspace of $\mathbb{M}_{n, m}(\mathbb{B}(\mathcal{H})) \cong \mathbb{B}\left(\mathcal{H}^{m}, \mathcal{H}^{n}\right)$. We allow to use the notation $\mathbb{M}_{I, J}(\mathbb{B}(\mathcal{H})) \cong \mathbb{B}\left(\mathcal{H}^{J}, \mathcal{H}^{I}\right)$ for arbitrary index sets $I$ and $J$. Let $X$ and $Y$ be operator spaces. The Haagerup tensor product of $X$ and $Y$ is the completion of the algebraic tensor product $X \otimes Y$ by the norm

$$
\begin{gathered}
\|u\|_{h}=\inf \left\{\left\|\left[a_{1}, \ldots, a_{n}\right]\right\|\left\|^{t}\left[b_{1}, \ldots, b_{n}\right]\right\| \mid u=\sum_{i=1}^{n} a_{i} \otimes b_{i} \in X \otimes Y,\right. \\
\left.n \in \mathbb{N}, a_{i} \in X, b_{i} \in Y\right\},
\end{gathered}
$$

and is denoted by $X \otimes_{h} Y$ [4]. We also recall the extended Haagerup tensor product $X \otimes_{e h} Y$. An element $u$ of $X \otimes_{e h} Y$ is represented by the following formal sum:

$$
u=\sum_{i \in I} a_{i} \otimes b_{i},
$$

where $a=\left[a_{i}\right]_{i \in I} \in \mathbb{M}_{1, I}(X), b={ }^{t}\left[b_{i}\right]_{i \in I} \in \mathbb{M}_{I, 1}(Y)$ (in other words,

$$
\|a\|=\left\|\sum_{i \in I} a_{i} a_{i}^{*}\right\|^{1 / 2}<\infty, \quad\|b\|=\left\|\sum_{i \in I} b_{i}^{*} b_{i}\right\|^{1 / 2}<\infty
$$

Received July 9, 2013; Revised October 23, 2013.

2010 Mathematics Subject Classification. Primary 46L06, 46L05, 47L25.

Key words and phrases. operator space, Haagerup tensor product, extended Haagerup tensor product, Schur product.

This work of the second author was partially supported by Grant-in-Aid for Scientific Research (C)22540220. 
for $a_{i} \in X$ and $\left.b_{i} \in Y\right)$. We appreciate this formal sum as the bilinear form on $X^{*} \times Y^{*}$ as follows:

$$
u(f, g)=\sum_{i \in I} f\left(a_{i}\right) g\left(b_{i}\right) \quad \text { for } f \in X^{*}, g \in Y^{*} .
$$

For this element $u \in X \otimes_{e h} Y$, its norm is defined by

$$
\|u\|_{e h}=\inf \left\{\|a\|\|b\| \mid u=\sum_{i \in I} a_{i} \otimes b_{i}, a \in \mathbb{M}_{1, I}(X), b \in \mathbb{M}_{I, 1}(Y)\right\} .
$$

Then we can realize $X \otimes_{e h} Y$ as a subspace of the dual operator space $\left(X^{*} \otimes_{h}\right.$ $\left.Y^{*}\right)^{*}([7],[8])$.

In [9], the authors studied the Schur product on $\mathbb{B}(\mathcal{H})$ and used the extended Haagerup tensor product to describe the property of Schur product maps. Effros and Ruan has shown that $X \otimes_{h} Y$ is (completely isometrically) embedded to $X \otimes_{e h} Y$ [8]. We will be concerned with the difference between the Haagerup tensor product and the extended Haagerup tensor product, since it is essential to deal with Schur product maps derived from (possibly unbounded) operators. The Schur product map on $\mathbb{B}\left(\ell^{2}\right)$ is a normal $\ell^{\infty}$-bimodule map, where $\ell^{\infty}$ is a maximal abelian subalgebra of $\mathbb{B}\left(\ell^{2}\right)$ and is identified with the bounded sequences on $\mathbb{N}$ (c.f. [9]). As a deep result concerning (normal) bimodule maps, we often refer to the following theorem by Blecher and Smith in [3]: if $M$ is a von Neumann algebra, then $M \otimes_{w^{*} h} M$ is completely isomorphic to the completely bounded $M^{\prime}$-bimodule maps of $\mathbb{K}(\mathcal{H})$ to $\mathbb{B}(\mathcal{H})$ denoted by $C B_{M^{\prime}}(\mathbb{K}(\mathcal{H}), \mathbb{B}(\mathcal{H}))$, where $\otimes_{w^{*} h}$ coincides with $\otimes_{e h}$ in this setting.

In Section 2, we study the difference between $\ell^{\infty} \otimes_{h} \ell^{\infty}$ and $\ell^{\infty} \otimes_{e h} \ell^{\infty}$ from the view point of Schur product and characterize them in terms of Schur product maps. Moreover we characterize $c_{0} \otimes_{h} c_{0}$ and $c_{0} \otimes_{e h} c_{0}$ in terms of Schur product maps, where $c_{0}$ is the complex sequences on $\mathbb{N}$ tends to 0 . As a result for Schur product maps derived from bounded operators, we show that $\ell^{\infty} \otimes_{h} \ell^{\infty} \cap \mathbb{B}\left(\ell^{2}\right)$ (resp. $\left.\quad \ell^{\infty} \otimes_{e h} \ell^{\infty} \cap \mathbb{B}\left(\ell^{2}\right)\right)$ coincides with $c_{0} \otimes_{h} c_{0} \cap \mathbb{B}\left(\ell^{2}\right)$ (resp. $c_{0} \otimes_{e h} c_{0} \cap \mathbb{B}\left(\ell^{2}\right)$ ).

In Section 3, we introduce some notions (right-compact, weakly right-compact, left-compact, weakly left-compact) for which distinguish the Haagerup tensor product from the extended Haagerup tensor product for operator spaces. As a main result in this section, for $\mathrm{C}^{*}$-algebras $A, B$, it is shown that $A \otimes_{h} B=$ $A \otimes_{e h} B$ if and only if $A$ or $B$ is finite-dimensional.

\section{2. $\ell^{\infty} \otimes_{h} \ell^{\infty}$ and $\ell^{\infty} \otimes_{e h} \ell^{\infty}$}

Let $X$ and $Y$ be operator spaces and $X \otimes Y$ the algebraic tensor product of $X$ and $Y$. For $a=\left[a_{1}, \ldots, a_{n}\right] \in \mathbb{M}_{1, n}(X), b={ }^{t}\left[b_{1}, \ldots, b_{n}\right] \in \mathbb{M}_{n, 1}(Y)$ and $\alpha=\left[\alpha_{i j}\right] \in \mathbb{M}_{n}(\mathbb{C})$, we denote $\sum_{i=1}^{n} a_{i} \otimes b_{i} \in X \otimes Y$ by $a \odot b$, and $\sum_{i, j=1}^{n} \alpha_{i j} a_{i} \otimes b_{j}$ by $a \alpha \odot b$. 
ON THE EXTENDED HAAGERUP TENSOR PRODUCT IN OPERATOR SPACES 347

Proposition 2.1. If $u \in X \otimes Y$, then

$$
\begin{aligned}
& \|u\|_{h}=\inf \{\|a\|\|\alpha\|\|b\| \mid u=a \alpha \odot b \in X \otimes Y, \\
& \left.\qquad n \in \mathbb{N}, \alpha \in \mathbb{M}_{n}(\mathbb{C}), a \in \mathbb{M}_{1, n}(X), b \in \mathbb{M}_{n, 1}(Y)\right\} .
\end{aligned}
$$

Proof. It follows from

$$
\begin{aligned}
\|u\|_{h} & =\inf \left\{\|a\|\left\|1_{n}\right\|\|b\| \mid u=a 1_{n} \odot b\right\} \\
& \geq \inf \{\|a\|\|\alpha\|\|b\| \mid u=a \alpha \odot b\} \\
& \geq \inf \{\|a \alpha\|\|b\| \mid u=a \alpha \odot b\} \\
& \geq \inf \{\|a\|\|b\| \mid u=a \odot b\}=\|u\|_{h} .
\end{aligned}
$$

For $\alpha=\left[\alpha_{i j}\right]_{i, j=1}^{\infty} \in \mathbb{B}\left(\ell^{2}\right), a=\left[a_{1}, a_{2}, \ldots\right] \in \mathbb{M}_{1, \infty}(X)$ and $b={ }^{t}\left[b_{1}, b_{2}, \ldots\right] \in$ $\mathbb{M}_{\infty, 1}(Y)$, we set

$$
\begin{gathered}
\alpha(k)=\left[\alpha_{i j}\right]_{i, j=1}^{k} \in \mathbb{M}_{k}(\mathbb{C}), \quad a(k)=\left[a_{1}, a_{2}, \ldots, a_{k}\right] \in \mathbb{M}_{1, k}(X) \\
\text { and } b(k)={ }^{t}\left[b_{1}, b_{2}, \ldots, b_{k}\right] \in \mathbb{M}_{k, 1}(Y)
\end{gathered}
$$

for $k=1,2,3, \ldots$ If the sequence $\{\alpha(k) a(k) \odot b(k)\}_{k=1}^{\infty}$ becomes a Cauchy sequence in $X \otimes_{h} Y$, then we denote this limit by

$$
a \alpha \otimes b \in X \otimes_{h} Y .
$$

When $\alpha$ belongs to $\mathbb{K}\left(\ell^{2}\right)$, we can see $a \alpha \otimes b$ as the usual limit of convergent sequences

$$
\lim _{k \rightarrow \infty} \sum_{i, j=1}^{k} \alpha_{i, j} a_{i} \otimes b_{j}=\lim _{n, m \rightarrow \infty} \sum_{i=1}^{n} \sum_{j=1}^{m} \alpha_{i j} a_{i} \otimes b_{j}
$$

in $X \otimes_{h} Y$ by the following reason. We choose a finite subset $J(k)$ of $\{(i, j) \mid$ $\max \{i, j\}>k\}$, then we have

$$
\left\|\sum_{(i, j) \in J(k)} \alpha_{i j} a_{i} \otimes b_{j}\right\|_{h}=\|a(N) \beta \odot b(N)\|_{h} \leq\|\beta\|\|a\|\|b\|,
$$

where $N=\max \{i, j \mid(i, j) \in J(k)\}, \beta=\left[\beta_{i j}\right]_{i, j=1}^{N} \in \mathbb{M}_{N}(\mathbb{C})$ and

$$
\beta_{i j}= \begin{cases}0 & (i, j) \notin J(k) \\ \alpha_{i j} & (i, j) \in J(k) .\end{cases}
$$

If we choose a sufficiently large $k$, then we can make $\left\|\sum_{(i, j) \in J(k)} \alpha_{i j} a_{i} \otimes b_{j}\right\|_{h}$ sufficiently small because of the compactness of $\alpha$.

Proposition 2.2. If $u \in X \otimes_{h} Y$, then

$$
\begin{gathered}
\|u\|_{h}=\inf \left\{\|a\|\|\alpha\|\|b\| \mid \alpha \in \mathbb{K}\left(\ell^{2}\right), a \in \mathbb{M}_{1, \infty}(X), b \in \mathbb{M}_{\infty, 1}(Y),\right. \\
\left.u=a \alpha \otimes b=\sum_{i, j=1}^{\infty} \alpha_{i j} a_{i} \otimes b_{j}\right\} \\
=\inf \left\{\max _{i}\left|\lambda_{i}\right|\|a\|\|b\| \mid\left(\lambda_{i}\right) \in c_{0}, a \in \mathbb{M}_{1, \infty}(X), b \in \mathbb{M}_{\infty, 1}(Y),\right.
\end{gathered}
$$




$$
\left.u=\sum_{i=1}^{\infty} \lambda_{i} a_{i} \otimes b_{i}\right\}
$$

Proof. Suppose that $u \in X \otimes_{h} Y$ with $\|u\|_{h}<1$. To prove the first equality, it suffices to show that there exist $a=\left[a_{1}, a_{2}, \ldots\right] \in \mathbb{M}_{1, \infty}(X)$ with $\|a\|<1$, $\alpha=\left[\alpha_{i j}\right] \in \mathbb{K}\left(\ell^{2}\right)$ with $\|\alpha\|<1$ and $b={ }^{t}\left[b_{1}, b_{2}, \ldots\right] \in \mathbb{M}_{\infty, 1}(Y)$ with $\|b\|<1$ such that

$$
\sum_{i, j=1}^{k} \alpha_{i j} a_{i} \otimes b_{j}
$$

converges to $u$ in $X \otimes_{h} Y$ when $k$ tends to $\infty$.

Given $\varepsilon=1-\|u\|_{h}>0$. Then we can choose a sequence $\left\{u_{n}\right\} \subset X \otimes Y$, which converges to $u$, satisfying that $\left\|u_{n}\right\|_{h}<1-\varepsilon$ and $\left\|u_{n+1}-u_{n}\right\|_{h}<2^{-n} \varepsilon$ $(n \geq 1), u_{0}=0$. If we put $t_{n}=u_{n+1}-u_{n}$, then it turns out

$$
\left\|\sum_{n=0}^{k} t_{n}-u\right\|_{h}=\left\|u_{k+1}-u\right\|_{h} \rightarrow 0(k \rightarrow \infty) .
$$

For $t_{n} \in X \otimes Y$, there exist $v_{n} \in \mathbb{M}_{1, \ell(n)}(X), \beta_{n} \in \mathbb{M}_{\ell(n)}$ and $w_{n} \in \mathbb{M}_{\ell(n), 1}$ such that $t_{n}=v_{n} \beta_{n} \otimes w_{n}$ with $\left\|\beta_{n}\right\|=1(n \geq 0),\left\|v_{n}\right\|\left\|w_{n}\right\|<2^{-n} \varepsilon(n \geq 1)$, $\left\|v_{0}\right\|\left\|w_{0}\right\|<1-\varepsilon$ and $\left\|v_{n}\right\|=\left\|w_{n}\right\|$. It follows that

$$
\sum_{n=0}^{\infty}\left\|t_{n}\right\|_{h} \leq \sum_{n=0}^{\infty}\left\|v_{n}\right\|\left\|w_{n}\right\|<1 .
$$

Then we can choose an increasing sequence $\left\{c_{n}\right\} \subset \mathbb{R}$ such that

$$
c_{n}>1, \lim _{n \rightarrow \infty} c_{n}=\infty, \sum_{n=0}^{\infty} c_{n}\left\|v_{n}\right\|\left\|w_{n}\right\|<1 .
$$

Now we put $a(i)=\sqrt{c}_{i} v_{i}, \alpha_{i}=\beta_{i} / c_{i}$ and $b(i)=\sqrt{c_{i}} w_{i}$. Then we have

$$
\begin{aligned}
u_{k+1} & =\sum_{n=0}^{k} v_{n} \beta_{n} \odot w_{n}=\sum_{n=0}^{k} a(n) \alpha_{n} \odot b(n) \\
& =\left[\begin{array}{llll}
a(0) & a(1) & \ldots & a(k)
\end{array}\right]\left[\begin{array}{llll}
\alpha_{0} & & \\
& \alpha_{1} & & \\
& & \ddots & \\
& & & \alpha_{k}
\end{array}\right] \odot\left[\begin{array}{c}
b(0) \\
b(1) \\
\vdots \\
b(k)
\end{array}\right],
\end{aligned}
$$

and $\|[a(0), a(1), \ldots, a(k)]\|,\left\|^{t}[b(0), b(1), \ldots, b(k)]\right\|<1,\left\|\alpha_{k}\right\| \rightarrow 0(k \rightarrow \infty)$. If we define $a_{n} \in X, b_{n} \in Y$ and $\alpha \in \mathbb{K}\left(\ell^{2}\right)$ by the following relation:

$$
\begin{aligned}
{[a(0), a(1), \ldots, a(k)] } & =\left[a_{1}, a_{2}, \ldots, a_{\ell(0)+\ell(1)+\cdots+\ell(k)}\right] \\
{[b(0), b(1), \ldots, b(k)] } & =\left[b_{1}, b_{2}, \ldots, b_{\ell(0)+\ell(1)+\cdots+\ell(k)}\right] \\
\alpha & =\bigoplus_{k=0}^{\infty} \alpha_{k},
\end{aligned}
$$


then we can get the first equality.

For the above $\alpha \in \mathbb{K}\left(\ell^{2}\right)$, we can take unitaries $u_{k}, v_{k} \in \mathbb{M}_{\ell(k)}$ such that

$$
\alpha_{k}=u_{k}\left[\begin{array}{llll}
\lambda_{\sum_{i=0}^{k-1} \ell(i)+1} & & & \\
& \lambda_{\sum_{i=0}^{k-1} \ell(i)+2} & & \\
& & \ddots & \\
& & & \lambda_{\sum_{i=0}^{k} \ell} \ell(i)
\end{array}\right] v_{k} \quad(k=0,1,2, \ldots) .
$$

If we put

$$
U=\bigoplus_{k=0}^{\infty} u_{k}, V=\bigoplus_{k=0}^{\infty} v_{k}, \Lambda=\left[\begin{array}{llll}
\lambda_{1} & & & \\
& \lambda_{2} & & \\
& & \lambda_{3} & \\
& & & \ddots
\end{array}\right]
$$

then we can get

$$
\begin{gathered}
\|\Lambda\|=\max _{i}\left|\lambda_{i}\right|, \\
a U \in \mathbb{M}_{1, \infty}(X) \text { and }\|a\|=\|a U\|, \\
V b \in \mathbb{M}_{\infty, 1}(Y) \text { and }\|b\|=\|V b\|,
\end{gathered}
$$

for any $a \in \mathbb{M}_{1, \infty}(X)$ and $b \in \mathbb{M}_{\infty, 1}(Y)$. By the fact

$$
a \alpha \otimes b=a U \Lambda V \otimes b=(a U) \Lambda \otimes(V b),
$$

we can get the second equality.

By the above proof, we also get the following fact:

$$
\begin{aligned}
X \otimes_{h} Y & =\left\{a \alpha \otimes b \mid \alpha \in \mathbb{K}\left(\ell^{2}\right), a \in \mathbb{M}_{1, \infty}(X), b \in \mathbb{M}_{\infty, 1}(Y)\right\} \\
& =\left\{\sum_{i=1}^{\infty} \lambda_{i} a_{i} \otimes b_{i} \mid\left(\lambda_{i}\right) \in c_{0}, a \in \mathbb{M}_{1, \infty}(X), b \in \mathbb{M}_{\infty, 1}(Y)\right\} .
\end{aligned}
$$

Let $\mathcal{H}$ be a separable Hilbert space, $\left\{f_{i}\right\}_{i=1}^{\infty}$ a completely orthonormal system of $\mathcal{H}$ and $\left\{e_{i j}\right\}_{i, j=1}^{\infty}$ a system of matrix units of $\mathbb{B}(\mathcal{H})$ defined by

$$
e_{i j} \xi=\left(\xi \mid f_{j}\right) f_{i}, \quad \xi \in \mathcal{H} .
$$

We can naturally identify the bounded sequences $\ell^{\infty}$ on $\mathbb{N}$ with the maximal abelian subalgebra of $\mathbb{B}(\mathcal{H})$ generated by $\left\{e_{i i}\right\}_{i=1}^{\infty}$. We denote by

$$
C B_{\ell \infty}(\mathbb{K}(\mathcal{H}), \mathbb{B}(\mathcal{H}))
$$

the $\ell^{\infty}$-bimodule completely bounded maps of $\mathbb{K}(\mathcal{H})$ to $\mathbb{B}(\mathcal{H})$. Then there exists completely isometric isomorphism between $\ell^{\infty} \otimes_{e h} \ell^{\infty}$ and $C B_{\ell \infty}(\mathbb{K}(\mathcal{H}), \mathbb{B}(\mathcal{H}))$ by the following: for $\sum_{i} a_{i} \otimes b_{i} \in \ell^{\infty} \otimes_{e h} \ell^{\infty},\left\langle\sum_{i} a_{i} \otimes b_{i}\right\rangle \in C B_{\ell \infty}(\mathbb{K}(\mathcal{H}), \mathbb{B}(\mathcal{H}))$ is defined by

$$
\left\langle\sum_{i} a_{i} \otimes b_{i}\right\rangle(k)=\sum_{i} a_{i} k b_{i}
$$


for $k \in \mathbb{K}(\mathcal{H})[3]$. By the $\ell^{\infty}$-bimodularity of $\langle x\rangle$ for $x \in \ell^{\infty} \otimes_{e h} \ell^{\infty}$, there exists a scalar $x_{i j}$ satisfying that

$$
\langle x\rangle\left(e_{i j}\right)=x_{i j} e_{i j}, \quad i, j=1,2, \ldots
$$

Then we can define an infinite dimensional matrix

$$
[x]=\left[x_{i j}\right]_{i, j=1}^{\infty},
$$

and also identify $[x]$ with a linear map from $c_{c}(\mathbb{N})$ to $\ell^{\infty}$ as follows: for $\xi=$ $\left[\xi_{1}, \xi_{2}, \ldots\right] \in c_{c}(\mathbb{N})$

$$
[x] \xi=\left[\sum_{j=1}^{\infty} x_{1 j} \xi_{j}, \sum_{j=1}^{\infty} x_{2 j} \xi_{j}, \ldots\right],
$$

where $\xi=\left[\xi_{1}, \xi_{2}, \ldots\right] \in c_{c}(\mathbb{N})$ means that $\xi_{n}=0$ for sufficiently large $n$. Clearly $c_{c}(\mathbb{N})$ is contained in $\ell^{2}$ and the image of $c_{c}(\mathbb{N})$ by $[x]$ is not necessarily contained in $\ell^{2}$. If $[x]$ can be extended to $\mathbb{B}\left(\ell^{2}\right)$ (resp. $\mathbb{K}\left(\ell^{2}\right)$ ), then we write $x \in\left(\ell^{\infty} \otimes_{e h} \ell^{\infty}\right) \cap \mathbb{B}$ (resp. $\left.x \in\left(\ell^{\infty} \otimes_{e h} \ell^{\infty}\right) \cap \mathbb{K}\right)$. We also use the following notation: for any subspace $S$ of $\ell^{\infty} \otimes_{e h} \ell^{\infty}$,

$$
\begin{aligned}
& S \cap \mathbb{B}=\left(\ell^{\infty} \otimes_{e h} \ell^{\infty}\right) \cap \mathbb{B} \cap S, \\
& S \cap \mathbb{K}=\left(\ell^{\infty} \otimes_{e h} \ell^{\infty}\right) \cap \mathbb{K} \cap S .
\end{aligned}
$$

Lemma 2.3. $x \in \ell^{\infty} \otimes_{h} \ell^{\infty}$ if and only if there exist $\beta \in \mathbb{K}\left(\ell^{2}\right), \xi_{i}, \eta_{i} \in \ell^{2}$ $(i=1,2, \ldots)$ such that

$$
\sup _{i}\left\{\left\|\xi_{i}\right\|,\left\|\eta_{i}\right\|\right\}<\infty \text { and } x_{i j}=\left(\beta \xi_{i} \mid \eta_{j}\right) .
$$

Proof. By Proposition 2.2, for given $\varepsilon>0$ and $x \in \ell^{\infty} \otimes_{h} \ell^{\infty}$, there exist $\left[a_{1}, a_{2}, \ldots\right] \in \mathbb{M}_{1, \infty}\left(\ell^{\infty}\right),{ }^{t}\left[b_{1}, b_{2}, \ldots\right] \in \mathbb{M}_{\infty, 1}\left(\ell^{\infty}\right)$ and $\left[\alpha_{i j}\right] \in \mathbb{K}(\mathcal{H})$ satisfying $\left\|\left[a_{1}, a_{2}, \ldots\right]\right\|\left\|t\left[b_{1}, b_{2}, \ldots\right]\right\|<1$ and $\left\|\left[\alpha_{i j}\right]\right\|<\|x\|_{h}+\varepsilon$ such that $x=\sum_{i, j=1}^{\infty} \alpha_{i j} a_{i} \otimes b_{j}$. If $\xi_{i}=\left[a_{1}(i), a_{2}(i), \ldots\right], \eta_{i}=\left[\overline{b_{1}(i)}, \overline{b_{2}(i)}, \ldots\right]$ and $\beta=\left[\beta_{i j}\right]$ where $\beta_{i j}=\alpha_{j i}$, then it is clear that $\sup _{i}\left\{\left\|\xi_{i}\right\|,\left\|\eta_{i}\right\|\right\}<\infty$. Thus we have

$$
\begin{aligned}
\langle x\rangle\left(e_{i j}\right) & =\sum_{s, t} \alpha_{s t} a_{s} e_{i j} b_{t} \\
& =\sum_{s, t} \alpha_{s t} a_{s}(i) b_{t}(j) e_{i j} \\
& =\left(\beta \xi_{i} \mid \eta_{j}\right) e_{i j} .
\end{aligned}
$$

Conversely, for given $\xi_{i}=\left[\xi_{i}(1), \xi_{i}(2), \ldots\right], \eta_{i}=\left[\eta_{i}(1), \eta_{i}(2), \ldots\right] \in \ell^{2}$ and $\beta=\left[\beta_{i j}\right] \in \mathbb{K}\left(\ell^{2}\right)$, we put $a_{i}=\left[\xi_{1}(i), \xi_{2}(i), \ldots\right], b_{i}=\left[\overline{\eta_{1}(i)}, \overline{\eta_{2}(i)}, \ldots\right] \in \ell^{\infty}$ and $\alpha=\left[\alpha_{i j}\right] \in \mathbb{K}\left(\ell^{2}\right)$ where $\alpha_{i j}=\beta_{j i}$. Then we have, for any positive integer $N$, $\left\|\left[a_{1}, a_{2}, \ldots, a_{N}\right]\right\|,\left\|^{t}\left[b_{1}, b_{2}, \ldots, b_{N}\right]\right\| \leq \sup \left\{\left\|\xi_{i}\right\|,\left\|\eta_{i}\right\|\right\}<\infty$. 
For an element

$$
x_{n}=\left[\begin{array}{llll}
a_{1} & a_{2} & \ldots & a_{n}
\end{array}\right]\left[\begin{array}{ccc}
\alpha_{11} & \cdots & \alpha_{1 n} \\
\vdots & \ddots & \vdots \\
\alpha_{n 1} & \cdots & \alpha_{n n}
\end{array}\right] \odot\left[\begin{array}{c}
b_{1} \\
b_{2} \\
\vdots \\
b_{n}
\end{array}\right] \in \ell^{\infty} \otimes \ell^{\infty}
$$

we have

$$
\begin{aligned}
x_{n+k}-x_{n}= & {\left[\begin{array}{cccccc}
a_{1} & \ldots & a_{n+k}
\end{array}\right] \times } \\
& {\left[\begin{array}{cccccc}
0 & \cdots & 0 & \alpha_{1, n+1} & \ldots & \alpha_{1, n+k} \\
\vdots & & \vdots & \vdots & & \vdots \\
0 & \cdots & 0 & \vdots & & \vdots \\
\alpha_{n+1,1} & \cdots & \cdots & \alpha_{n+1, n+1} & & \vdots \\
\vdots & & & & \ddots & \vdots \\
\alpha_{n+k, 1} & \cdots & \cdots & \ldots & \cdots & \alpha_{n+k, n+k}
\end{array}\right] \odot\left[\begin{array}{c}
b_{1} \\
\vdots \\
b_{n+k}
\end{array}\right] . }
\end{aligned}
$$

By the compactness of $\alpha$ and Proposition 2.1,

$$
\lim _{n \rightarrow \infty}\left\|x_{n+k}-x_{n}\right\|_{h}=0
$$

for any positive integer $k$. Thus we have that the sequence $\left\{x_{n}\right\}$ converges to $x$ in $\ell^{\infty} \otimes_{h} \ell^{\infty}$.

Since $c_{0}$ is a $C^{*}$-subalgebra of $\ell^{\infty}$, we can see $c_{0} \otimes_{h} c_{0}$ as a subspace of $\ell^{\infty} \otimes_{h} \ell^{\infty}$.

Lemma 2.4. $x \in c_{0} \otimes_{h} c_{0}$ if and only if there exist $\xi_{i}, \eta_{i} \in \ell^{2}(i=1,2, \ldots)$ such that

$$
\lim _{i}\left\|\xi_{i}\right\|=\lim _{i}\left\|\eta_{i}\right\|=0 \text { and } x_{i j}=\left(\xi_{i} \mid \eta_{j}\right) .
$$

Proof. By Proposition 2.2, for given $\varepsilon>0$ and $x \in c_{0} \otimes_{h} c_{0}$, there exist $\left[a_{1}, a_{2}, \ldots\right] \in \mathbb{M}_{1, \infty}\left(c_{0}\right),{ }^{t}\left[b_{1}, b_{2}, \ldots\right] \in \mathbb{M}_{\infty, 1}\left(c_{0}\right)$ and $\left[\alpha_{i j}\right] \in \mathbb{K}(\mathcal{H})$ satisfying $\left\|\left[a_{1}, a_{2}, \ldots\right]\right\|\left\|^{t}\left[b_{1}, b_{2}, \ldots\right]\right\|<1$ and $\left\|\left[\alpha_{i j}\right]\right\|<\|x\|_{h}+\varepsilon$ such that $x=\sum_{i, j=1}^{\infty} \alpha_{i j} a_{i} \otimes b_{j}$. We put $\xi_{i}=\left[a_{1}(i), a_{2}(i), \ldots\right], \eta_{i}=\left[\overline{b_{1}(i)}, \overline{b_{2}(i)}, \ldots\right]$ and $\beta=\left[\beta_{i j}\right]$ where $\beta_{i j}=\alpha_{j i}$. Then we have

$$
x_{i j}=\left(\beta \xi_{i} \mid \eta_{j}\right)
$$

and, by the fact $a_{i}, b_{i} \in c_{0}$,

$$
\lim _{i \rightarrow \infty} \xi_{i}(j)=\lim _{i \rightarrow \infty} \eta_{i}(j)=0 \text { for any } j \in \mathbb{N} .
$$

This means that $\left\{\xi_{i}\right\},\left\{\eta_{i}\right\} \subset \ell^{2}$ weakly converge to 0 . We can choose $\beta_{1}, \beta_{2}$ $\in \mathbb{K}\left(\ell^{2}\right)$ such that $\beta=\beta_{2}^{*} \beta_{1}$. Then we have

$$
\lim _{i}\left\|\beta_{1} \xi_{i}\right\|=\lim _{i}\left\|\beta_{2} \eta_{i}\right\|=0
$$


and

$$
x_{i j}=\left(\beta \xi_{i} \mid \eta_{j}\right)=\left(\beta_{1} \xi_{i} \mid \beta_{2} \eta_{j}\right) .
$$

Conversely suppose that $\lim _{i \rightarrow \infty}\left\|\xi_{i}\right\|=\lim _{i \rightarrow \infty}\left\|\eta_{i}\right\|=0$. We may assume that $\left\|\xi_{i}\right\|<c$ for all $i \in \mathbb{N}$. Then, for any $\varepsilon>0$, we can choose a number $N$ such that

$$
\left\|\xi_{i}^{\prime}\right\|<c \text { for all } i \text { and }\left\|\xi_{i}^{\prime}\right\|<\varepsilon \text { if } i>N
$$

where

$$
\xi_{i}^{\prime}(j)= \begin{cases}\xi_{i}(j) & \text { if } j<N \\ 2 \xi_{i}(j) & \text { otherwise. }\end{cases}
$$

Clearly we have $\lim _{i \rightarrow \infty}\left\|\xi_{i}^{\prime}\right\|=0$. Applying this argument to $\left\{\xi_{i}\right\}$ repeatedly, we can choose $1=n(0)<n(1)<n(2)<\cdots$ and $\left\{\zeta_{i}\right\} \subset \ell^{2}$ such that

$$
\begin{gathered}
\zeta_{i}(j)=2^{k} \xi_{i}(j) \quad \text { if } n(k) \leq j<n(k+1), \\
\left\|\zeta_{i}\right\|<c \text { for all } i \text { and }\left\|\zeta_{i}\right\|<2^{-k} \text { if } i>n(k) .
\end{gathered}
$$

We put $a_{i}=\left[\zeta_{1}(i), \zeta_{2}(i), \ldots\right], b_{i}=\left[\overline{\eta_{1}(i)}, \overline{\eta_{2}(i)}, \ldots\right]$ and

$$
\lambda_{i}=2^{-k} \quad \text { if } n(k) \leq i<n(k+1) .
$$

Then we have $a_{i}, b_{i},\left(\lambda_{i}\right) \in c_{0}$, and

$$
\left\|\left[a_{1}, a_{2}, \ldots\right]\right\|,\left\|^{t}\left[b_{1}, b_{2}, \ldots\right]\right\| \leq \sup \left\{\left\|\zeta_{i}\right\|,\left\|\eta_{i}\right\|\right\}<\infty .
$$

Thus we have

$$
x=\sum_{i=1}^{\infty} \lambda_{i} a_{i} \otimes b_{i} \in c_{0} \otimes_{h} c_{0} .
$$

Combining these lemmas, we can get the following fact:

Theorem 2.5. (1) For $x \in \ell^{\infty} \otimes_{h} \ell^{\infty}$,

$$
\|x\|_{h}=\inf \left\{\sup _{i j}\left\|\xi_{i}\right\|\left\|\eta_{j}\right\|\|\beta\| \mid x_{i j}=\left(\beta \xi_{i} \mid \eta_{j}\right), \xi_{i}, \eta_{j} \in \ell^{2}, \beta \in \mathbb{K}\left(\ell^{2}\right)\right\} .
$$

(2) For $x \in c_{0} \otimes_{h} c_{0}$,

$\|x\|_{h}=\inf \left\{\sup _{i j}\left\|\xi_{i}\right\|\left\|\eta_{j}\right\| \mid x_{i j}=\left(\xi_{i} \mid \eta_{j}\right), \xi_{i}, \eta_{j} \in \ell^{2}, \xi_{i} \rightarrow 0, \eta_{j} \rightarrow 0\right.$ strongly $\}$.

The correspondence between Schur multiplier and the tensor product with a suitable norm is known as in [12], and the result is obtained by Spronk in [15]. By using the extended Haagerup norm on $\ell^{\infty} \otimes_{e h} \ell^{\infty}$ and $c_{0} \otimes_{e h} c_{0}$, we can rewrite them as follows:

Proposition 2.6 ([15], Corollary 3.2). (1) For $x \in \ell^{\infty} \otimes_{e h} \ell^{\infty}$,

$$
\|x\|_{e h}=\inf \left\{\sup _{i j}\left\|\xi_{i}\right\|\left\|\eta_{j}\right\| \mid x_{i j}=\left(\xi_{i} \mid \eta_{j}\right), \xi_{i}, \eta_{j} \in \ell^{2}\right\} .
$$

(2) For $x \in c_{0} \otimes_{e h} c_{0}$,

$\|x\|_{e h}=\inf \left\{\sup _{i j}\left\|\xi_{i}\right\|\left\|\eta_{j}\right\| \mid x_{i j}=\left(\xi_{i} \mid \eta_{j}\right), \xi_{i}, \eta_{j} \in \ell^{2}, \xi_{i} \rightarrow 0, \eta_{j} \rightarrow 0\right.$ weakly $\}$. 
ON THE EXTENDED HAAGERUP TENSOR PRODUCT IN OPERATOR SPACES 353

Theorem 2.7. (1) $\left(c_{0} \otimes_{h} c_{0}\right) \cap \mathbb{B}=\left(\ell^{\infty} \otimes_{h} \ell^{\infty}\right) \cap \mathbb{B}$.

(2) $\left(c_{0} \otimes_{e h} c_{0}\right) \cap \mathbb{B}=\left(\ell^{\infty} \otimes_{e h} \ell^{\infty}\right) \cap \mathbb{B}$.

Proof. (1) It is clear that $\left(c_{0} \otimes_{h} c_{0}\right) \cap \mathbb{B} \subset\left(\ell^{\infty} \otimes_{h} \ell^{\infty}\right) \cap \mathbb{B}$.

Let $x \in\left(\ell^{\infty} \otimes_{h} \ell^{\infty}\right) \cap \mathbb{B}$. By Lemma 2.3 , there exist $\beta \in \mathbb{K}\left(\ell^{2}\right), \xi_{i}, \eta_{i} \in \ell^{2}$ $(i=1,2, \ldots)$ such that

$$
\sup _{i}\left\{\left\|\xi_{i}\right\|,\left\|\eta_{i}\right\|\right\}<\infty \text { and } x_{i j}=\left(\beta \xi_{i} \mid \eta_{j}\right) .
$$

We choose $\beta_{1}, \beta_{2} \in \mathbb{K}\left(\ell^{2}\right)$ such that $\beta=\beta_{2}^{*} \beta_{1}$, that is,

$$
x_{i j}=\left(\beta_{1} \xi_{i} \mid \beta_{2} \eta_{j}\right) \text {, }
$$

and we may assume that

$$
\operatorname{Range}\left(\beta_{1}\right) \subset \overline{\operatorname{span}\left\{\beta_{2} \eta_{j} \mid j \in \mathbb{N}\right\}}
$$

and

$$
\operatorname{Range}\left(\beta_{2}\right) \subset \overline{\operatorname{span}\left\{\beta_{1} \xi_{j} \mid j \in \mathbb{N}\right\}} .
$$

It is sufficient to show that

$$
\lim _{i}\left\|\beta_{1} \xi_{i}\right\|=\lim _{i}\left\|\beta_{2} \eta_{i}\right\|=0 .
$$

Assume that

$$
\limsup _{i}\left\|\beta_{1} \xi_{i}\right\|>0 .
$$

Then there exist $\delta>0$ and a subsequence $\{n(k)\}$ such that $\left\|\beta_{1} \xi_{n(k)}\right\|>\delta$ for $k=1,2, \ldots$. Since $\sup \left\|\xi_{i}\right\|<\infty$, we may also assume that $\left\{\xi_{n(k)}\right\}$ weakly converges to some $\xi_{0} \in \ell^{2}$. By the compactness of $\beta_{1}$, we have

$$
\lim _{i}\left\|\beta_{1} \xi_{n(k)}-\beta_{1} \xi_{0}\right\|=0 .
$$

Thus it turns out $\beta_{1} \xi_{0} \neq 0$. We can choose $j_{0}$ such that

$$
\left(\beta_{1} \xi_{0} \mid \beta_{2} \eta_{j_{0}}\right) \neq 0 \text {. }
$$

Then there exists $K \in \mathbb{N}$ such that

$$
\left|x_{n(k), j_{0}}\right|=\left|\left(\beta_{1} \xi_{n(k)} \mid \beta_{2} \eta_{j_{0}}\right)\right|>\frac{\left|\left(\beta_{1} \xi_{0} \mid \beta_{2} \eta_{j_{0}}\right)\right|}{2} \quad \text { for } k>K .
$$

This contradicts to $[x]=\left[x_{i j}\right] \in \mathbb{B}\left(\ell^{2}\right)$.

(2) For $x \in\left(\ell^{\infty} \otimes_{e h} \ell^{\infty}\right) \cap \mathbb{B}$, that is, $[x] \in \mathbb{B}\left(\ell^{2}\right)$, we can choose $\alpha=\left[\xi_{i j}\right]$ and $\beta=\left[\eta_{i j}\right]$ in $\mathbb{B}\left(\ell^{2}\right)$ such that

$$
[x]=\alpha \beta \text { and }\|\alpha\|=\|\beta\|=\|[x]\|^{1 / 2} .
$$

Remarking the fact

$$
x_{i j}=\sum_{k} \xi_{i k} \eta_{k j}
$$

we define

$$
a_{i}=\left[\xi_{1 i}, \xi_{2 i}, \ldots\right], b_{i}=\left[\eta_{i 1}, \eta_{i 2}, \ldots\right] \in \ell^{2} \subset c_{0}
$$


for all $i$. Then we have

$$
\left\|\left[a_{1}, a_{2}, \ldots\right]\right\|,\left\|^{t}\left[b_{1}, b_{2}, \ldots\right]\right\| \leq\|[x]\|^{1 / 2}<\infty
$$

and $x=\sum_{i} a_{i} \otimes b_{i} \in c_{0} \otimes_{e h} c_{0}$.

Corollary 2.8. Let $x \in \ell^{\infty} \otimes_{e h} \ell^{\infty}$ and

$$
\limsup _{k}\left|x_{i(k), j(k)}\right|>0
$$

for some injection $\mathbb{N} \ni k \rightarrow(i(k), j(k)) \in \mathbb{N} \times \mathbb{N}$. Then $x$ does not belong to $c_{0} \otimes_{h} c_{0}$.

Moreover, if $x$ satisfies an additional condition $[x] \in \mathbb{B}\left(\ell^{2}\right)$, then $x$ does not belong to $\ell^{\infty} \otimes_{h} \ell^{\infty}$.

Example 2.9. (1) Let $x=\sum_{i, j=1}^{\infty}\left(\frac{\lambda_{i}}{\lambda_{j}}\right)^{\sqrt{-1} t} e_{i} \otimes e_{j} \in \ell^{\infty} \otimes_{e h} \ell^{\infty}$, where $\lambda_{i}$ 's are positive real and $t$ is real. Then we have

$$
\begin{gathered}
{[x]=\left[\begin{array}{ccc}
\left(\frac{\lambda_{1}}{\lambda_{1}}\right)^{\sqrt{-1} t} & \left(\frac{\lambda_{1}}{\lambda_{2}}\right)^{\sqrt{-1} t} & \ldots . \\
\left(\frac{\lambda_{2}}{\lambda_{1}}\right)^{\sqrt{-1} t} & \left(\frac{\lambda_{2}}{\lambda_{2}}\right)^{\sqrt{-1} t} & \ldots \\
\vdots & \vdots & \ddots
\end{array}\right] \notin \mathbb{B}\left(\ell^{2}\right),} \\
x_{i j}=\left(\frac{\lambda_{i}}{\lambda_{j}}\right)^{\sqrt{-1} t}=\left(\left[\begin{array}{ccc}
1 & & \\
& 0 & \\
& & \ddots
\end{array}\right]\left[\begin{array}{c}
\lambda_{i}^{\sqrt{-1}} t \\
0 \\
\vdots
\end{array}\right] \mid\left[\begin{array}{c}
\lambda_{j}^{\sqrt{-1}} t \\
0 \\
\vdots
\end{array}\right]\right)
\end{gathered}
$$

and $\left|x_{i j}\right|=1$. This means $x \notin\left(\ell^{\infty} \otimes_{e h} \ell^{\infty}\right) \cap \mathbb{B}, x \notin c_{0} \otimes_{e h} c_{0}$ (by Proposition 2.6) and $x \in \ell^{\infty} \otimes_{h} \ell^{\infty}$ (by Lemma 2.3).

(2) Let $x=\sum_{k=1}^{\infty} e_{k} \otimes e_{k} \in c_{0} \otimes_{e h} c_{0}$. Since

$$
[x]=\left[\begin{array}{ccc}
1 & 0 & \cdots \\
0 & 1 & \cdots \\
\vdots & \vdots & \ddots
\end{array}\right] \in \mathbb{B}\left(\ell^{2}\right)
$$

then we have $x \notin \ell^{\infty} \otimes_{h} \ell^{\infty}$ (by Corollary 2.8).

(3) $\left(\ell^{\infty} \otimes_{e h} \ell^{\infty}\right) \cap \mathbb{K} \varsubsetneqq\left(\ell^{\infty} \otimes_{h} \ell^{\infty}\right) \cap \mathbb{B}$.

By Lemma 2.3, it is clear that $\left(\ell^{\infty} \otimes_{e h} \ell^{\infty}\right) \cap \mathbb{K} \subset\left(\ell^{\infty} \otimes_{h} \ell^{\infty}\right) \cap \mathbb{B}$.

We consider the following infinite dimensional matrix:

$$
p=\left[\begin{array}{ccccccccc}
1 & & & & & & & \\
& \frac{1}{2} & \frac{1}{2} & & & & & \\
& \frac{1}{2} & \frac{1}{2} & & & & & \\
& & & \frac{1}{3} & \frac{1}{3} & \frac{1}{3} & & \\
& & \frac{1}{3} & \frac{1}{3} & \frac{1}{3} & & \\
& & \frac{1}{3} & \frac{1}{3} & \frac{1}{3} & & \\
& & & & & \ddots & \\
& & & & & & \ddots
\end{array}\right] .
$$


ON THE EXTENDED HAAGERUP TENSOR PRODUCT IN OPERATOR SPACES 355

Since $p$ is an infinite dimensional projection, $p$ does not belong to $\mathbb{K}\left(\ell^{2}\right)$. If we put

$$
\begin{gathered}
\xi_{1}=[1,0,0,0, \ldots] \\
\xi_{2}=\xi_{3}=\left[0, \frac{1}{\sqrt{2}}, 0,0 \ldots\right] \\
\xi_{4}=\xi_{5}=\xi_{6}=\left[0,0,0, \frac{1}{\sqrt{3}}, 0, \ldots\right]
\end{gathered}
$$

and $\xi_{n}=\eta_{n}(n=1,2, \ldots)$, then $\xi_{n}, \eta_{n} \in \ell^{2}$ satisfy

$$
\lim _{n}\left\|\xi_{n}\right\|=\lim _{n}\left\|\eta_{n}\right\|=0 \text { and } p=\left[\left(\xi_{i} \mid \eta_{j}\right)\right] .
$$

This means that

$$
\left(\left(\ell^{\infty} \otimes_{h} \ell^{\infty}\right) \cap \mathbb{B}\right) \cap\left(\left(\ell^{\infty} \otimes_{e h} \ell^{\infty}\right) \cap \mathbb{K}\right)^{c} \neq \phi .
$$

(4) Let $a=b=\left[1, \frac{1}{\sqrt{2}}, \ldots, \frac{1}{\sqrt{n}}, \ldots\right] \in c_{0}$. Then $x=a \otimes b \in c_{0} \otimes_{h} c_{0}$ and

$$
\left[x_{i j}\right]=\left[\begin{array}{ccc}
1 & \frac{1}{\sqrt{2}} & \cdots \\
\frac{1}{\sqrt{2}} & \frac{1}{2} & \cdots \\
\vdots & \vdots & \ddots
\end{array}\right] \notin \mathbb{B}\left(\ell^{2}\right) .
$$

By the above argument, we can get the following diagram of inclusions:

$$
\begin{array}{cccc}
\left(c_{0} \otimes_{h} c_{0}\right) \cap \mathbb{K} & \varsubsetneqq \quad\left(c_{0} \otimes_{h} c_{0}\right) \cap \mathbb{B} & \varsubsetneqq & \left(c_{0} \otimes_{e h} c_{0}\right) \cap \mathbb{B} \\
& \left(\ell^{\infty} \otimes_{h} \ell^{\infty}\right) \cap \mathbb{B} & \varsubsetneqq & \left(\ell^{\infty} \otimes_{e h} \ell^{\infty}\right) \cap \mathbb{B} \\
c_{0} \otimes_{h} c_{0} & \varsubsetneqq & c_{0} \otimes_{e h} c_{0} \\
\ell^{\infty} \otimes_{h} \ell^{\infty} & \varsubsetneqq & \ell^{\infty} \otimes_{e h} \ell^{\infty}
\end{array}
$$

\section{3. $X \otimes_{h} Y$ and $X \otimes_{e h} Y$}

Let $X$ be an operator space. We call $X$ is right-compact (resp. left-compact) if $\mathbb{M}_{1, I}(X)=\mathbb{M}_{1, I}(X) \mathbb{K}\left(\ell^{2}(I)\right)$ (resp. $\left.\mathbb{M}_{I, 1}(X)=\mathbb{K}\left(\ell^{2}(I)\right) \mathbb{M}_{I, 1}(X)\right)$. If $X$ is right-compact, then, for any $a=\left[a_{i}\right]_{i \in I} \in \mathbb{M}_{1, I}(X)$, there exist $b=\left[b_{i}\right]_{i \in I} \in$ $\mathbb{M}_{1, I}(X)$ and $\alpha=\left[\alpha_{i j}\right]_{i, j \in I} \in \mathbb{K}\left(\ell^{2}(I)\right)$ such that

$$
a=b \alpha \quad\left(a_{j}=\sum_{i \in I} b_{i} \alpha_{i j}\right) .
$$

We also call $X$ is weakly right-compact (resp. weakly left-compact) if we have, for any $a=\left[a_{i}\right] \in \mathbb{M}_{1, I}(X)$ (resp. $a=\left[a_{i}\right] \in \mathbb{M}_{I, 1}(X)$ ), that $\left\{i \in I \mid a_{i} \neq 0\right\}$ is countable and $\lim _{i \rightarrow \infty}\left\|a_{i}\right\|=0$. 
Lemma 3.1. If $X$ is a right-compact (resp. left-compact) operator space, then $X$ is weakly right-compact (resp. weakly left-compact).

Proof. Since $\alpha=\left[\alpha_{i j}\right]_{i, j \in I} \in \mathbb{K}\left(\ell^{2}(I)\right)$, we have that $\left\{(i, j) \in I \times I \mid \alpha_{i j} \neq 0\right\}$ is countable and $\left\{i \in I \mid a_{i} \neq 0\right\}$ is also countable. So we may assume that $I=\mathbb{N}$. Then we have $a_{i}=\sum_{j=1}^{\infty} b_{j} \alpha_{j i}$ and

$$
\begin{aligned}
\left\|a_{i}\right\| & =\left\|\left[\begin{array}{lll}
b_{1} & b_{2} & \cdots
\end{array}\right]\left[\begin{array}{c}
\alpha_{1 i} \\
\alpha_{2 i} \\
\vdots
\end{array}\right]\right\| \leq\left\|\left[\begin{array}{lll}
b_{1} & b_{2} & \cdots
\end{array}\right]\right\|\left\|\left[\begin{array}{c}
\alpha_{1 i} \\
\alpha_{2 i} \\
\vdots
\end{array}\right]\right\| \\
& =\left\|\sum_{j=1}^{\infty}\left|\alpha_{j i}\right|^{2} \mid\right\|^{1 / 2}\|\| \sum_{i=1}^{\infty} b_{i} b_{i}^{*} \|^{1 / 2} .
\end{aligned}
$$

By the assumtion, we have

$$
\left\|\sum_{i=1}^{\infty} b_{i} b_{i}^{*}\right\|<\infty \text { and } \lim _{i \rightarrow \infty} \sum_{j=1}^{\infty}\left|\alpha_{j i}\right|^{2}=0 .
$$

This means that

$$
\lim _{i \rightarrow \infty}\left\|a_{i}\right\|=0
$$

As a typical example of right-compact operator spaces, we can get the following:

Lemma 3.2. Let $X$ be an operator space on a Hilbert space $\mathcal{H}$. If $X \subset p \mathbb{B}(\mathcal{H})$ for some finite-dimensional projection $p \in \mathbb{B}(\mathcal{H})$, then $X$ is right-compact.

In particular, any finite-dimensional $C^{*}$-algebra is left- and right-compact.

Proof. We assume that $\operatorname{dim} p \mathcal{H}=n<\infty$. Let $a=\left[a_{i}\right]_{i \in I} \in \mathbb{M}_{1, I}(X)$, i.e.,

$$
\left\|\sum_{i \in I} a_{i} a_{i}^{*}\right\|<\infty .
$$

We can consider $a_{i} a_{i}^{*}$ as an element of $\mathbb{M}_{n}(\mathbb{C})$, so we put $a_{i} a_{i}^{*}=\left(\alpha_{j k}^{i}\right)(j, k=$ $1,2, \ldots, n)$. By the positivity of $a_{i} a_{i}^{*}$, we have

$$
0 \leq \max _{1 \leq j \leq n} \sum_{i \in I} \alpha_{j j}^{i} \leq\left\|\sum_{i \in I} a_{i} a_{i}^{*}\right\|
$$

This implies that

$$
I_{0}=\left\{i \in I \mid \alpha_{j j}^{i}>0 \text { for some } j\right\}
$$

is countable, so we may assume $I=\mathbb{N}$. Remarking the fact

$$
\left\|a_{i} a_{i}^{*}\right\| \leq \sqrt{n} \max _{1 \leq j \leq n} \alpha_{j j}^{i}
$$

we have

$$
\left\|\sum_{i=1}^{\infty} a_{i} a_{i}^{*}\right\| \leq \sum_{i=1}^{\infty}\left\|a_{i} a_{i}^{*}\right\| \leq \sqrt{n} \sum_{i=1}^{\infty} \max _{1 \leq j \leq n} \alpha_{j j}^{i} \leq \sqrt{n} \sum_{i=1}^{\infty} \sum_{j=1}^{n} \alpha_{j j}^{i}
$$


ON THE EXTENDED HAAGERUP TENSOR PRODUCT IN OPERATOR SPACES 357

$$
=\sqrt{n} \sum_{j=1}^{n} \sum_{i=1}^{\infty} \alpha_{j j}^{i} \leq n \sqrt{n} \max _{1 \leq j \leq n} \sum_{i=1}^{\infty} \alpha_{j j}^{i}<\infty .
$$

We can choose numbers $0=\lambda_{0}<\lambda_{1}<\lambda_{2}<\cdots$ such that

$$
\left\|\sum_{i=\lambda_{k}+1}^{\infty} a_{i} a_{i}^{*}\right\|<\frac{1}{2^{k}}\left\|\sum_{i=1}^{\infty} a_{i} a_{i}^{*}\right\|
$$

and set $b_{i}=\sqrt{k} a_{i}\left(\lambda_{k-1}<i \leq \lambda_{k}\right)$. Since

$$
\left\|\sum_{i=1}^{\infty} b_{i} b_{i}^{*}\right\|=\left\|\sum_{k=0}^{\infty} \sum_{i=\lambda_{k}+1}^{\infty} a_{i} a_{i}^{*}\right\|<\sum_{k=0}^{\infty} \frac{1}{2^{k}}\left\|\sum_{i=1}^{\infty} a_{i} a_{i}^{*}\right\|=2\left\|\sum_{i=1}^{\infty} a_{i} a_{i}^{*}\right\|<\infty,
$$

we have

$$
a=\left[a_{i}\right]_{i \in \mathbb{N}}=\left[b_{i}\right]_{i \in \mathbb{N}}\left[\frac{1}{\sqrt{k(i)}} \delta_{i j}\right]_{i, j \in \mathbb{N}} \in \mathbb{M}_{1 \mathbb{N}}(X) \mathbb{K}\left(\ell^{2}\right),
$$

where $k(i)=k$ if $\lambda_{k-1}<i \leq \lambda_{k}$ and $\delta_{i j}$ means Kronecker's symbol.

Lemma 3.3. Let $\left\{a_{i}\right\}_{i=1}^{\infty}$ be a sequence of bounded operators on a Hilbert space $\mathcal{H}$ with $\left\|\sum_{i=1}^{\infty} a_{i} a_{i}^{*}\right\|<\infty$. Suppose that there exist sequences $\left\{\xi_{i}\right\}_{i=1}^{\infty},\left\{\eta_{i}\right\}_{i=1}^{\infty}$ of unit vectors in $\mathcal{H}$ such that

$$
\left|\left(a_{i} \xi_{i} \mid \eta_{i}\right)\right|>1 \quad \text { for } i \in \mathbb{N} .
$$

Then, for any $\varepsilon>0$, there exists a number $i_{0}$ such that

$$
\left\{i \in \mathbb{N}||\left(a_{i_{0}} \xi_{i} \mid \eta_{i}\right) \mid<\varepsilon\right\}
$$

is infinite.

Proof. Let $\varepsilon>0$. Suppose that $\left\{j \in \mathbb{N}||\left(a_{i} \xi_{j} \mid \eta_{j}\right) \mid<\varepsilon\right\}$ is finite for all $i \in \mathbb{N}$. If we omit the finite set

$$
\left\{i \in \mathbb{N}||\left(a_{1} \xi_{i} \mid \eta_{i}\right) \mid<\varepsilon\right\}
$$

we may assume that

$$
\begin{gathered}
\left|\left(a_{1} \xi_{1} \mid \eta_{1}\right)\right|>1, \\
\left|\left(a_{2} \xi_{2} \mid \eta_{2}\right)\right|>1, \quad\left|\left(a_{1} \xi_{2} \mid \eta_{2}\right)\right| \geq \varepsilon .
\end{gathered}
$$

If we omit again the finite set

$$
\left\{i \in \mathbb{N}|i>2,|\left(a_{2} \xi_{i} \mid \eta_{i}\right) \mid<\varepsilon\right\},
$$

we may assume that

$$
\left|\left(a_{1} \xi_{3} \mid \eta_{3}\right)\right| \geq \varepsilon, \quad\left|\left(a_{2} \xi_{3} \mid \eta_{3}\right)\right| \geq \varepsilon .
$$

Using this argument repeatedly, we may assume that, for any $n$,

$$
\left|\left(a_{1} \xi_{n} \mid \eta_{n}\right)\right| \geq \varepsilon,\left|\left(a_{2} \xi_{n} \mid \eta_{n}\right)\right| \geq \varepsilon, \ldots,\left|\left(a_{n-1} \xi_{n} \mid \eta_{n}\right)\right| \geq \varepsilon .
$$


Then we have

$$
\begin{aligned}
(n-1) \varepsilon^{2} & \leq \sum_{i=1}^{n-1}\left|\left(a_{i} \xi_{n} \mid \eta_{n}\right)\right|^{2} \leq \sum_{i=1}^{n-1}\left\|\xi_{n}\right\|^{2}\left\|a_{i}^{*} \eta_{n}\right\|^{2} \\
& \leq \sum_{i=1}^{n-1}\left(a_{i} a_{i}^{*} \eta_{n} \mid \eta_{n}\right) \leq\left\|\sum_{i=1}^{n-1} a_{i} a_{i}^{*}\right\| .
\end{aligned}
$$

This contradicts to the assumption $\left\|\sum_{i=1}^{\infty} a_{i} a_{i}^{*}\right\|<\infty$. Therefore we can get a number $i_{0}$ required in the statement.

Lemma 3.4. Let $X$ be an operator space on a Hilbert space $\mathcal{H}$. If $X$ is not weakly right-compact, then there exist a sequence $\left\{a_{i}\right\}$ of $X$, sequences $\left\{\xi_{i}\right\}$, $\left\{\eta_{i}\right\}$ of unit vectors in $\mathcal{H}$ and some constant $K$ such that
(1) $\left\|\sum_{i=1}^{\infty} a_{i} a_{i}^{*}\right\|<\infty$.
(2) $3<\left\|a_{i}\right\|<K$.
(3) $3<\left|\left(a_{i} \xi_{i} \mid \eta_{i}\right)\right|<K$.
(4) $\left|\left(a_{k} \xi_{j} \mid \eta_{j}\right)\right| \leq \frac{1}{K^{k}}$ for $k \neq j$.

Proof. Since $X$ is not weakly right-compact, we can choose a sequence $\left\{a_{i}\right\}$ of $X$ such that

$$
\left\|\sum_{i=1}^{\infty} a_{i} a_{i}^{*}\right\|<\infty
$$

and $\left\{\left\|a_{i}\right\|\right\}$ is not convergent to 0 . Then we may assume that $3<\left\|a_{i}\right\|<K$ for any $i$ and some constant $K$. We choose sequences $\left\{\xi_{i}\right\},\left\{\eta_{i}\right\}$ of unit vectors in $\mathcal{H}$ satisfying

$$
\left|\left(a_{i} \xi_{i} \mid \eta_{i}\right)\right|>3 \quad \text { for all } i \in \mathbb{N} .
$$

Using Lemma 3.3, we can choose a subsequence $\{n(k)\}_{k=1}^{\infty}$ such that

$$
\left|\left(a_{n(k)} \xi_{n(j)} \mid \eta_{n(j)}\right)\right|<\frac{1}{K^{k}} \quad \text { for } k<j .
$$

If we replace $\left\{a_{n(k)}\right\}$ with $\left\{a_{i}\right\}$, then we can get the conditions (1)-(3) and (4) for $k<j$.

We consider a sequence $\left\{a_{i}, \xi_{i}, \eta_{i}\right\}$ of triplets. By the calculation

$$
\begin{aligned}
\sum_{i=1}^{\infty}\left|\left(a_{i} \xi_{j} \mid \eta_{j}\right)\right|^{2} & \leq \sum_{i=1}^{\infty}\left\|a_{i}^{*} \eta_{j}\right\|^{2}=\left(\sum_{i=1}^{\infty} a_{i} a_{i}^{*} \eta_{j} \mid \eta_{j}\right) \\
& \leq\left\|\sum_{i=1}^{\infty} a_{i} a_{i}^{*}\right\|<\infty
\end{aligned}
$$

we have

$$
\lim _{i \rightarrow \infty}\left|\left(a_{i} \xi_{j} \mid \eta_{j}\right)\right|=0 \quad \text { for any } j
$$


Choosing a subsequence of $\left\{a_{i}, \xi_{i}, \eta_{i}\right\}$, we may assume that

$$
\left|\left(a_{k} \xi_{j} \mid \eta_{j}\right)\right|<\frac{1}{K^{k}} \quad \text { for } k>j .
$$

Thus we can get the conditions (1)-(4).

Lemma 3.5. Let $\alpha>\beta>0$. If sequences $\left\{a_{k}\right\},\left\{b_{k}\right\}$ of vectors in $\mathbb{C}^{m}$ satisfy the following conditions:

$$
\left|\left(a_{k} \mid b_{k}\right)\right|>\alpha \text { and }\left|\left(a_{k} \mid b_{\ell}\right)\right|<\beta \text { for } k \neq \ell,
$$

then

$$
\sup \left\{\left|a_{k}(i)\right|,\left|b_{k}(i)\right| \mid i=1, \ldots, m, k=1,2, \ldots\right\}=\infty .
$$

Proof. We assume that $\sup \left\{\left|a_{k}(i)\right|,\left|b_{k}(i)\right| \mid i=1, \ldots, n, k=1,2, \ldots\right\}$ is finite. By the compactness, we can choose a pair of convergent subsequences $\left\{a_{n(k)}\right\},\left\{b_{n(k)}\right\}$. Then we have

$$
\lim _{k}\left|\left(a_{n(k)} \mid b_{n(k+1)}\right)\right|=\lim _{k}\left|\left(a_{n(k)} \mid b_{n(k)}\right)\right| \geq \alpha .
$$

But this contradicts to

$$
\lim \sup _{k}\left|\left(a_{n(k)} \mid b_{n(k+1)}\right)\right| \leq \beta .
$$

Theorem 3.6. Let $X$ and $Y$ be operator spaces. Then we have

(1) $X \otimes_{h} Y=X \otimes_{e h} Y$ if $X$ is right-compact or $Y$ is left-compact.

(2) $X$ is weakly right-compact or $Y$ is weakly left-compact if $X \otimes_{h} Y=$ $X \otimes_{e h} Y$.

Proof. (1) We assume that $X$ is right-compact. For any $s \in X \otimes_{e h} Y$, there exist $a=\left[a_{i}\right] \in \mathbb{M}_{1, I}(X)$ and $b={ }^{t}\left[b_{i}\right] \in \mathbb{M}_{I, 1}(Y)$ such that

$$
s=a \otimes b=\sum_{i \in I} a_{i} \otimes b_{i}
$$

By the assumption, there exist $c \in \mathbb{M}_{1, I}(X)$ and $\alpha \in \mathbb{K}\left(\ell^{2}(I)\right)$ such that $a=c \alpha$. So we have

$$
s=a \otimes b=c \alpha \otimes b \in X \otimes_{h} Y .
$$

This means that $X \otimes_{h} Y=X \otimes_{e h} Y$. When $Y$ is left-compact, we can also have $X \otimes_{h} Y=X \otimes_{e h} Y$ by the same argument.

(2) Let $X$ (resp. $Y$ ) be an operator space on $\mathcal{H}$ (resp. $\mathcal{K}$ ). We assume that $X$ is not weakly right-compact and $Y$ is not weakly left-compact. By Lemma 3.4 , we can choose a sequence $\left\{a_{i}\right\}$ of $X$ (resp. a sequence $\left\{b_{i}\right\}$ of $Y$ ), sequences $\left\{\xi_{i}\right\},\left\{\eta_{i}\right\}$ of unit vectors in $\mathcal{H}$ (resp. sequences $\left\{\tilde{\xi}_{i}\right\},\left\{\tilde{\eta}_{i}\right\}$ of unit vectors in $\mathcal{K}$ ) and some constant $K$ satisfying that

$$
\begin{gathered}
\left\|\sum_{i=1}^{\infty} a_{i} a_{i}^{*}\right\|<\infty,\left\|\sum_{i=1}^{\infty} b_{i}^{*} b_{i}\right\|<\infty, \\
3<\left\|a_{i}\right\|,\left\|b_{i}\right\|<K,
\end{gathered}
$$




$$
3<\left|\left(a_{i} \xi_{i} \mid \eta_{i}\right)\right|,\left|\left(b_{i} \tilde{\eta}_{i} \mid \tilde{\xi}_{i}\right)\right|<K
$$

and

$$
\left|\left(a_{k} \xi_{j} \mid \eta_{j}\right)\right|,\left|\left(b_{k} \tilde{\eta}_{j} \mid \tilde{\xi}_{j}\right)\right|<\frac{1}{K^{k}} \quad \text { for } k \neq j .
$$

We define $s \in X \otimes_{e h} Y, \varphi_{k} \in X^{*}$ and $\psi_{k} \in Y^{*}$ as follows:

$$
s=\sum_{i=1}^{\infty} a_{i} \otimes b_{i}, \quad \varphi_{k}(\cdot)=\left(\cdot \xi_{k} \mid \eta_{k}\right), \quad \psi_{k}(\cdot)=\left(\cdot \tilde{\eta_{k}} \mid \tilde{\xi_{k}}\right) .
$$

Then we have

$$
\begin{aligned}
\left|s\left(\varphi_{k}, \psi_{k}\right)\right| & =\left|\sum_{i=1}^{\infty} \varphi_{k}\left(a_{i}\right) \psi_{k}\left(b_{i}\right)\right| \\
& =\left|\sum_{i=1}^{\infty}\left(a_{i} \xi_{k} \mid \eta_{k}\right)\left(b_{i} \tilde{\eta_{k}} \mid \tilde{\xi_{k}}\right)\right| \\
& \geq\left|\left(a_{k} \xi_{k} \mid \eta_{k}\right)\left(b_{k} \tilde{\eta_{k}} \mid \tilde{\xi_{k}}\right)\right|-\sum_{i \neq k}\left|\left(a_{i} \xi_{k} \mid \eta_{k}\right)\left(b_{i} \tilde{\eta_{k}} \mid \tilde{\xi_{k}}\right)\right| \\
& \geq 9-\sum_{i=1}^{\infty} \frac{1}{K^{2 i}}>8,
\end{aligned}
$$

and, for $j \neq k$,

$$
\begin{aligned}
\left|s\left(\varphi_{j}, \psi_{k}\right)\right| & =\left|\sum_{i=1}^{\infty} \varphi_{j}\left(a_{i}\right) \psi_{k}\left(b_{i}\right)\right| \\
& =\left|\sum_{i=1}^{\infty}\left(a_{i} \xi_{j} \mid \eta_{j}\right)\left(b_{i} \tilde{\eta_{k}} \mid \tilde{\xi_{k}}\right)\right| \\
& \leq \sum_{i=1}^{\infty}\left|\left(a_{i} \xi_{j} \mid \eta_{j}\right)\left(b_{i} \tilde{\eta_{k}} \mid \tilde{\xi_{k}}\right)\right| \\
& \leq \frac{1}{K^{j-1}}+\frac{1}{K^{k-1}}+\sum_{i=1}^{\infty} \frac{1}{K^{2 i}}<3 .
\end{aligned}
$$

Suppose that $X \otimes_{e h} Y=X \otimes_{h} Y$, then $s$ belongs to $X \otimes_{h} Y$. We can choose

$$
t=\sum_{i=1}^{m} x_{i} \otimes y_{i} \in X \otimes_{h} Y \text { and }\|s-t\|_{h}<1 .
$$

Since $\left\|\varphi_{j}\right\|=\left\|\psi_{k}\right\|=1$,

$$
\left|s\left(\varphi_{j}, \psi_{k}\right)-t\left(\varphi_{j}, \psi_{k}\right)\right|<1,
$$

that is,

$$
\left|s\left(\varphi_{j}, \psi_{k}\right)-\sum_{i=1}^{m} \varphi_{j}\left(x_{i}\right) \psi_{k}\left(y_{i}\right)\right|<1 .
$$


Then we have

$$
\left|\sum_{i=1}^{m} \varphi_{k}\left(x_{i}\right) \psi_{k}\left(y_{i}\right)\right|>7
$$

and, for $j \neq k$,

$$
\left|\sum_{i=1}^{m} \varphi_{j}\left(x_{i}\right) \psi_{k}\left(y_{i}\right)\right|<4 .
$$

This contradicts to the boundedness of $\left\{\left|\varphi_{k}\left(x_{i}\right)\right|,\left|\psi_{k}\left(y_{i}\right)\right| \mid 1 \leq i \leq m, k \in \mathbb{N}\right\}$ by Lemma 3.5. We are done.

Remark 3.7. The row Hilbert space $\mathcal{H}_{r}$ is right-compact and is not weakly leftcompact and the column Hilbert space $\mathcal{H}_{c}$ is left-compact and is not weakly right-compact. Then it is clear that

$$
\mathcal{H}_{r} \otimes_{h} \mathcal{H}_{c}=\mathcal{H}_{r} \otimes_{e h} \mathcal{H}_{c}, \mathcal{H}_{c} \otimes_{h} \mathcal{H}_{r} \neq \mathcal{H}_{c} \otimes_{e h} \mathcal{H}_{r}
$$

(c.f. $[6])$.

Corollary 3.8. Let $A$ and $B$ be $C^{*}$-algebras. Then the following assertions are equivalent:

(1) $A \otimes_{h} B=A \otimes_{e h} B$,

(2) $A$ or $B$ is finite dimensional.

Proof. We have already shown that every finite-dimensinal $\mathrm{C}^{*}$-algebra is rightcompact and left-compact in Lemma 3.2. It is sufficient to show that every infinite-dimensional $\mathrm{C}^{*}$-algebra is neither weakly right-compact nor weakly leftcompact.

Suppose that $A$ is infinite dimensional. Since the maximal abelian *-subalgebras in $A$ is infinite dimensional, there exist self-adjoint elements $\left\{a_{n}\right\} \subset A$ such that $\left\|a_{n}\right\|=1$ and $a_{i} a_{j}=0$ if $i \neq j$. Then we have

$$
\left\|\sum_{i=1}^{\infty} a_{i}^{2}\right\|=\left\|\sum_{i=1}^{\infty} a_{i} a_{i}^{*}\right\|=\left\|\sum_{i=1}^{\infty} a_{i}^{*} a_{i}\right\|<\infty
$$

and $\left\{\left\|a_{i}\right\|\right\}$ does not converge to 0 . This means that $A$ is neither weakly rightcompact nor weakly left-compact.

Acknowledgement. The authors would like to thank the referee for his careful comments.

\section{References}

[1] D. P. Blecher and C. Le Merdy, Operator Algebras and Their Modules, London Math. Soc. Monogr. New Ser. 30, Oxford Univ. Press, Oxford, 2004.

[2] D. P. Blecher and V. I. Paulsen, Tensor products of operator spaces, J. Funct. Anal. 99 (1991), no. 2, 262-292.

[3] D. P. Blecher and R. R. Smith, The dual of the Haagerup tensor product, J. London Math. Soc. 45 (1992), no. 1, 126-144.

[4] E. G. Effros and A. Kishimoto, Module maps and Hochschild-Johnson cohomology, Indiana Univ. Math. J. 36 (1987), no. 2, 257-276. 
[5] E. G. Effros and Z.-J. Ruan, A new approach to operator spaces, Canad. Math. Bull. 34 (1991), no. 3, 329-337.

[6] _ Self-duality for the Haagerup tensor product and Hilbert space factorizations, J. Funct. Anal. 100 (1991), no. 2, 257-284.

[7] _ Operator spaces, J. London Math. Soc. Monogr. New Ser. 23, Oxford Univ. Press, New York, 2000.

[8] - Operator space tensor products and Hopf convolution algebras, J. Operator Theory 50 (2003), no. 1, 131-156.

[9] T. Itoh and M. Nagisa, Schur products and module maps on $B(\mathcal{H})$, Publ. Res. Inst. Math. Sci. 36 (2000), no. 2, 253-268.

[10] V. I. Paulsen, Completely Bounded Maps and Operator Algebras, Cambridge Stud. Adv. Math. 78, Cambridge Univ. Press, Cambridge, 2002.

[11] V. I. Paulsen and R. R. Smith, Diagonals in tensor products of operator algebras, Proc. Edinb. Math. Soc. 45 (2002), no. 3, 647-652.

[12] G. Pisier, Similarity Problems and Completely Bounded Maps, Lecture Notes in Math. 1618, Springer Verlag, 2001.

[13] Introduction to Operator Space Theory, London Math. Soc. Lecture Notes Ser. 294, Cambridge Univ. Press, Cambridge, 2003.

[14] R. R. Smith, Completely bounded module maps and the Haagerup tensor product, J. Funct. Anal. 102 (1991), no. 1, 156-175.

[15] N. Spronk, Measurable Schur multipliers and completely bounded multipliers of the Fourier algebras, Proc. London Math. Soc. 89 (2004), no. 1, 161-192.

TAKASHI ITOH

Department of Mathematics

GunMa UNIVERSITY

GUNMA 371-8510, JAPAN

E-mail address: itoh@gunma-u.ac.jp

Masaru NAGisa

Graduate School of Science

Chiba University

CHIBA 263-8522, JAPAN

E-mail address: nagisa@math.s.chiba-u.ac.jp 